\title{
PSEUDOANEURISMA GIGANTE DE ARTÉRIA ESPLÊNICA
}

\section{GIANT PSEUDOANEURYSM OF THE SPLENIC ARTERY}

\author{
Orlando Jorge Martins Torres, TCBC-MA ${ }^{1}$ \\ Olívia Maria Veloso Costa ${ }^{2}$ \\ José Aldemir Teixeira Nunes Junior ${ }^{3}$
}

\section{INTRODUÇÃO}

O aneurisma de artéria esplênica é o mais freqüente aneurisma de artérias viscerais. Sua ocorrência varia de $0,16 \%$ em uma autópsia não selecionada da população a 7,1\% em autópsias realizadas em pacientes com hipertensão porta por cirrose. Estes aneurismas e os pseudoaneurismas raramente excedem em $3 \mathrm{~cm}$ de diâmetro e maiores que $10 \mathrm{~cm}$ são excepcionais. Somente oito casos foram registrados na literatura ${ }^{1,2}$. O trauma contuso está provavelmente envolvido na formação do pseudoaneurisma. O retardo no aparecimento varia de oito dias a dois anos. A história de trauma, o tamanho do hematoma e a ausência de outros fatores associados sugerem o diagnóstico. O principal risco na evolução do pseudoaneurisma gigante de artéria esplênica é a ruptura, sendo indicado o tratamento cirúrgico ${ }^{2,3}$.

Pretendemos neste estudo apresentar um caso de pseudoaneurisma gigante de artéria esplênica tratado com sucesso por cirurgia.

\section{RELATO DO CASO}

Paciente do sexo masculino, 26 anos, deu entrada na disciplina de clínica cirúrgica III do Hospital Universitário Presidente Dutra da Universidade Federal do Maranhão (UFMA) apresentando sangramento digestivo alto e baixo há aproximadamente quatro meses. Apresentou ainda neste período dor, aumento de volume abdominal e dispnéia aos es- forços. Etilista, tabagista crônico e usuário de drogas ilícitas. Referia ainda história de trauma em região lombar há aproximadamente dois anos.

Ao exame físico apresentava estado geral e de nutrição regulares, sinais vitais normais, mucosas hipocoradas $(++/ 4+)$, ausência de linfonodomegalias. O abdome era plano, doloroso à palpação no epigástrio e hipocôndrio esquerdo.

Os exames laboratoriais revelaram: hematócrito $24,5 \%$; hemoglobina $8,2 \mathrm{~g} / \mathrm{dl}$; leucócitos $10.840 / \mathrm{mm}^{3}$. Demais exames laboratoriais estavam normais. Realizou endoscopia digestiva alta que estava normal. Na arteriografia esplâncnica do tronco celíaco, mesentérica superior e mesentérica inferior, e na arteriografia seletiva esplênica, foi observada a presença de pseudoaneurisma gigante de artéria esplênica distal com extravasamento de contraste. $\mathrm{O}$ pseudoaneurisma encontrava-se em contato íntimo com a parede do corpo e fundo gástricos exercendo compressão sobre estas estruturas (Figuras 1 e 2). Não foram observadas outras alterações.

Com o diagnóstico pré-operatório estabelecido, o paciente foi preparado para tratamento cirúrgico. A cirurgia consistiu em laparotomia mediana, identificação de formação expansiva localizada no pedículo esplênico, de aproximadamente $15 \mathrm{~cm}$ em seu maior diâmetro, em íntimo contato com o baço e a parede posterior gástrica. Realizada a seguir a liberação do estômago, ligadura dupla e secção individual da artéria e veia esplênica proximal, liberação e dissecção dos ligamentos esplênicos com deslocamento do baço do seu leito. Retirada em monobloco do

1. Professor Livre-Docente e Coordenador da Disciplina de Clínica Cirúrgica III - UFMA.

2. Estudante de Medicina - UFMA.

3. Médico Radiologista Intervencionista do Hospital Universitário Presidente Dutra - UFMA.

Trabalho realizado na Disciplina de Clínica Cirúrgica III-Universidade Federal do Maranhão (UFMA). 


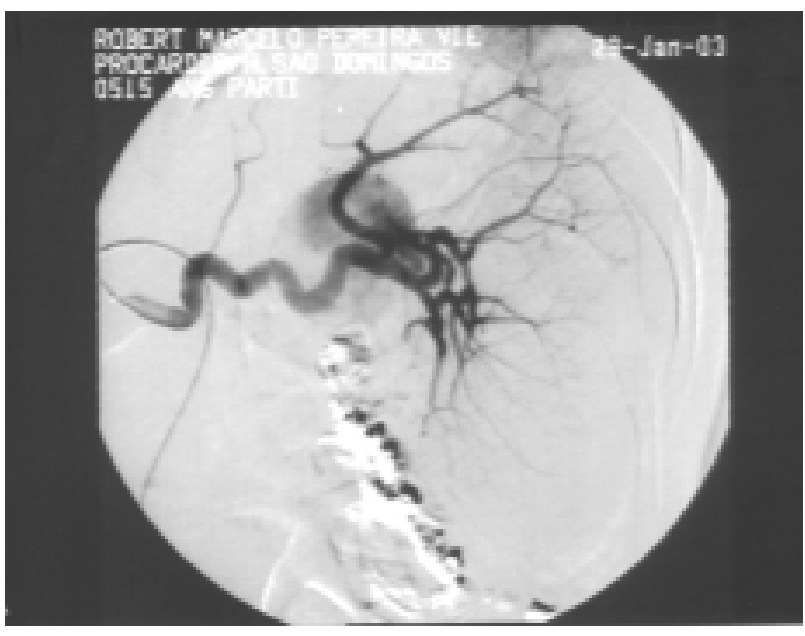

Figura 1 - Arteriografia seletiva esplênica (fase precoce). Opacificação parcial de grande formação aneurismática a partir de um ramo arterial segmentar.

pseudoaneurisma. Revisão da hemostasia e síntese da ferida operatória por planos.

Paciente permaneceu internado em unidade de terapia intensiva por dois dias, evoluindo sem complicações e recebeu alta hospitalar no $13^{\circ}$ dia do período pós-operatório sem intercorrências.

\section{DISCUSSÃO}

Os aneurismas e pseudoaneurismas gigantes da artéria esplênica são incomuns, com apenas oito casos anteriores descritos na literatura ${ }^{2}$. A sua etiologia e patogênese não são diferentes dos aneurismas menores. Dois fatores que contribuem para a etiologia e patogênese foram reconhecidos: a fraqueza preliminar da parede arterial e o aumento concomitante da pressão sanguínea. O trauma contuso associado à fraqueza da parede tem sido um fator importante na etiopatogênese do pseudoaneurisma de artéria esplênica ${ }^{2,3}$. No presente estudo o paciente era usuário de drogas e apresentava história de trauma abdominal há aproximadamente um ano.

O pseudoaneurisma gigante raramente é assintomático. Aclínica consiste em dor crônica, vaga, no epigástrio e hipocôndrio esquerdo. Hemorragia digestiva maciça por fístula gástrica tem sido registrado na literatura ${ }^{2,4}$. O sopro, presente ocasionalmente, origina-se da turbulência do fluxo aórtico. Um achado importante é a presença, na palpação, de uma massa pulsátil no quadrante superior esquerdo e epigástrio ${ }^{2,5}$.

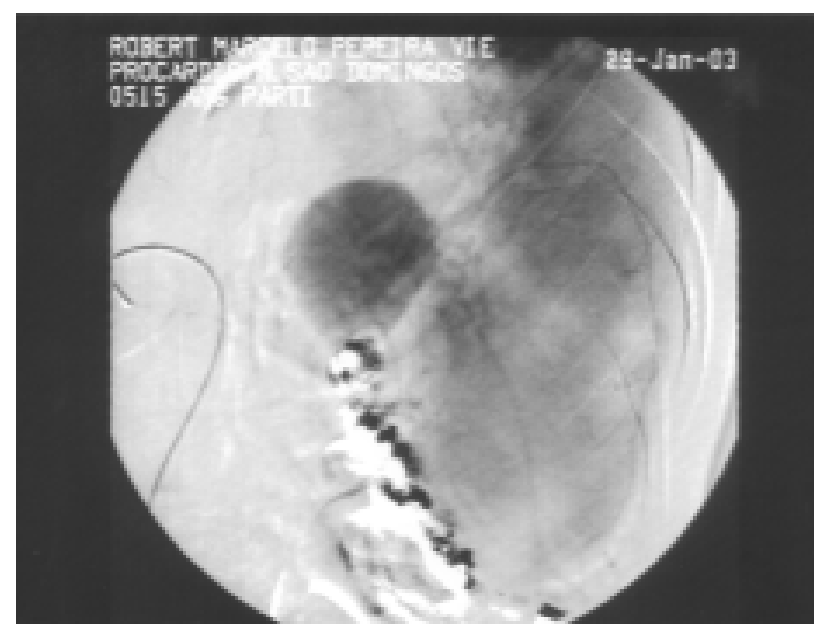

Figura 2 - Arteriografia seletiva esplênica (fase tardia). Opacificação completa de grande formação aneurismática esplênica.

O diagnóstico é confirmado pelo eco-Doppler com injeção de contraste em bolus. Este exame detecta a massa aneurismática com aderências a outros órgãos adjacentes e doenças associadas. A arteriografia global é necessária por diferentes razões: a) mostra a vascularização do pseudoaneurisma, b) evidencia anomalias vasculares, c) investiga a etiologia como as displasias musculares, d) pode delinear outros aneurismas viscerais ${ }^{1,2,5}$. No presente caso a arteriografia demonstrou o pseudoaneurisma e sua relação com o estômago e baço.

A ruptura do pseudoaneurisma é uma condição temida e sua freqüência é de 3 a $10 \%$ para os pseudoaneurismas pequenos e de $28 \%$ para aqueles gigantes. É caracterizado por dor abdominal aguda no epigástrio e hipocôndrio esquerdo associado a colapso cardiovascular. Aruptura durante a cirurgia também tem sido descrita ${ }^{1,4,5}$.

A finalidade do tratamento cirúrgico é a exclusão do pseudoaneurisma por clampeamento vascular. A abordagem deve ser, em princípio, por laparotomia, necessitando da abertura do grande omento com controle do tronco celíaco e da aorta supracelíaca. A toracofrenolaparotomia pode ser necessária para mais fácil mobilização da víscera abdominal. $\mathrm{O}$ baço deve ser preservado sempre que possível pelas complicações bem conhecidas da esplenectomia ${ }^{1,2,5}$. Neste caso realizamos o acesso abdominal, controle do pedículo vascular e liberação completa do baço, que foi removido em monobloco. Em nosso paciente não foi possível a preservação 
deste órgão o que foi observado em dois de seis casos descritos para aneurismas gigantes ${ }^{1,2}$.

A embolização da artéria esplênica previne a necessidade de cirurgia de emergência e proporciona um tratamento alternativo para pacientes com risco cirúrgico elevado. Esta tem sido descrita como o procedimento de escolha para o futuro quando superadas as potenciais complicações como embolização inadvertida de outros vasos e hemorragia intra-abdominal $2,3,5$.

\begin{abstract}
Splenic artery pseudoaneurysm larger than $10 \mathrm{~cm}$ is a rare condition. The risk of rupture is probably high and surgical treatment is necessary. The objective of this article is to report a case of a patient with giant pseudoaneurysm of the splenic artery submitted to surgical resection. A 26-year-old man complaining of gastrointestinal hemorrhage and abdominal pain The patient's medical history revealed that one year before he had an abdominal blunt trauma. The angiography showed a giant pseudoaneurysm of the splenic artery with compression of the stomach. The patient was operated on by abdominal access and the spleen and pseudoaneurysm were resected. The postoperative course was uneventful and the patient was discharged 13 days after surgery without problems.
\end{abstract}

Key Words: Aneurysm, false; splenic artery; Surgery.

\section{REFERÊNCIAS}

1. Mattar SG, Lumsden AB - The management of splenic artery aneurysms: experience with 23 cases. Am J Surg 1995, 169(6):580-584.

2. Bornet P, Medjoubi SA, Tissot A, et al. - Giant aneurysm of splenic artery: a case report. Angiology, 2000, 51(4):343-347.

3. Nortosky MC, Rogers FB, Shackford JR - Delayed presentation of splenic artery pseudoaneurysms following blunt abdominal trauma: case reports. J Trauma, 1995, 38(3):444-447.
4. Nakazawa T, Kida M, Kokubu S, et al. - Massive gastrointestinal hemorrhage from pseudocyst with fistula and splenic artery aneurysm rupture. Endoscopy, 1999, 31(3):S22-S23.

5. Long CD, Bakshi KR, Kahn MB, et al. - Giant splenic artery aneurysm. Ann Vasc Surg, 1993, 7(5):474-478.

Endereço para correspondência:

Dr. Orlando Torres

Rua dos Bicudos No 14 - Ed. Aspen - apto 600

Renascença II - CEP: 65075-090 - São Luís - MA

E-mail: otorres@elo.com.br 